\title{
ChemComm
}

\section{Development of a fluorescent sensor for an illicit date rape drug - GBL $†$}

Cite this: Chem. Commun., 2013 49,6170

Received 29th April 2013, Accepted 22nd May 2013

DOI: $10.1039 / c 3 c c 43153 c$

www.rsc.org/chemcomm

The first fluorescent sensor for an illicit date rape drug, GBL, was developed and named Green Date. It shows high fluorescence enhancement to GBL and allows its detection in different drinks. The mechanism between GBL and Green Date was explored. This discovery may help to prevent the drug-facilitated sexual assault problems.

Drug-facilitated sexual assault (DFSA) is a criminal act, which is defined as the "voluntary or involuntary ingestion of a drug by a victim that results in an act of sexual activity without consent". ${ }^{1}$ The drugs that can be used to assist in the execution of DFSA are called "date rape drugs", including alcohol, gamma-hydroxybutyric acid (GHB), gamma-butyrolactone (GBL), Rohypnol, ketamine, and Soma. ${ }^{2}$ In addition to alcohol, GHB and GBL are the most commonly used date rape drugs. GHB is a powerful depressant for the central nervous system and is used illicitly for its sedative and euphoric effects. ${ }^{3}$ It was banned for sale as a supplement in US by FDA in 1990. ${ }^{4}$ As a result of the more stringent regulations to purchase GHB, consumers began to turn to its pro-drug, GBL. ${ }^{5}$ GBL itself is pharmacologically inactive, however, it is easily metabolized to GHB in the presence of peripheral lactonases in vivo (Fig. 1). ${ }^{6}$ Therefore, GHB and GBL have similar psychopharmacological effects after ingestion. GBL is a common industrial solvent and its usage in the legitimate chemical industry is approved, which makes its availability even spread in the internet. ${ }^{7}$ Both GHB and GBL are notorious daterape drugs due to their speedy elimination after ingestion, colourless property and high solubility in aqueous solutions. ${ }^{8}$ In contrast to GHB, GBL is more lipophilic and can be absorbed upon oral administration more rapidly, leading to its higher bioavailability. ${ }^{9}$ Overdose of GBL may lead to dangerously low respiratory rates, unconsciousness, seizures, bradycardia and even death. ${ }^{10}$

\footnotetext{
${ }^{a}$ Department of Chemistry and MedChem Program, Life Sciences Institute, National University of Singapore, 3 Science Drive 3, Singapore 117543. E-mail: chmcyt@nus.edu.sg

${ }^{b}$ Laboratory of Bioimaging Probe Development, Singapore Bioimaging Consortium (SBIC), Agency for Science, Technology and Research ( ${ }^{*}$ STAR), Biopolis,

11 Biopolis Way, \#02-02 Helios, Singapore 138667

† Electronic supplementary information (ESI) available: Detailed synthetic and screening procedures, characterization data, additional spectroscopic information and details of beverages. See DOI: 10.1039/c3cc43153c
}

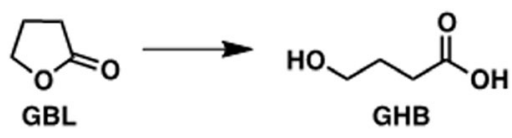

Fig. 1 Structures of $\mathrm{GBL}$ and $\mathrm{GHB}$.

Efforts have been made over the years to develop detection kits for these date-rape drugs. Several test kits have been introduced in the market, including "DrinkSafe ${ }^{\mathrm{TM}}$ ", cards, "DrinkSafe ${ }^{\mathrm{TM}}$ ", coasters $^{11}$ and "Drink Detective ${ }^{\mathrm{TM}}$ ". ${ }^{12}$ Another recent development in such a kit for date rape drugs is "drug detection straw", ${ }^{13}$ developed by Tel Aviv University in 2011. However, all these kits are only applicable for the detection of GHB, ketamine or Rohypnol. No on-site detection method has been reported for GBL yet. Its less harsh legal status, low cost and easier availability have made GBL a more preferred date rape drug over GHB. ${ }^{14}$ Development of a real time detection method for GBL would be a great contribution to prevention of the DFSA. Here, we report the first development of a fluorescent sensor for the illicit date rape drug - GBL.

The diversity-oriented fluorescence library approach (DOFLA) has proved its ability in sensor development in the last few decades. ${ }^{15}$ For novel GBL sensor discovery, we designed a high throughput image based screening system. Pictures of DOFL compounds before and after addition of GBL were taken and their intensities compared (Fig. 2 and Fig. S1, ESI $\dagger$ ). The sedative dosage of GBL is between 2 and 3 grams per ingestion. ${ }^{16}$ As the average volume of a drink is between 150 and $200 \mathrm{~mL}$, the minimum concentration of GBL in a spiked beverage would be $10 \mathrm{mg} \mathrm{mL} \mathrm{m}^{-1}$. Therefore, the screening concentration of GBL was set to $10 \mathrm{mg} \mathrm{mL}{ }^{-1}$ in water. After screening of 5120 dyes generated from different fluorescent scaffolds, 83 compounds (data not shown) were selected as primary hits due to their fluorescence intensity change from the pictures. Secondary screening was then carried out on a fluorescent microplate reader with a wide range of concentrations of GBL (i.e., 3, 5, $10,20,40 \mathrm{mg} \mathrm{mL}^{-1}$ ) to eliminate the false positive results using a 96-well black plate. Finally, 5 best responsive and reproducible hit compounds were rendered (Fig. S3, ESI $\dagger$ ).

These 5 hit compounds belong to our previously reported BODIPY library BDD, ${ }^{17}$ and all these compounds share structural 


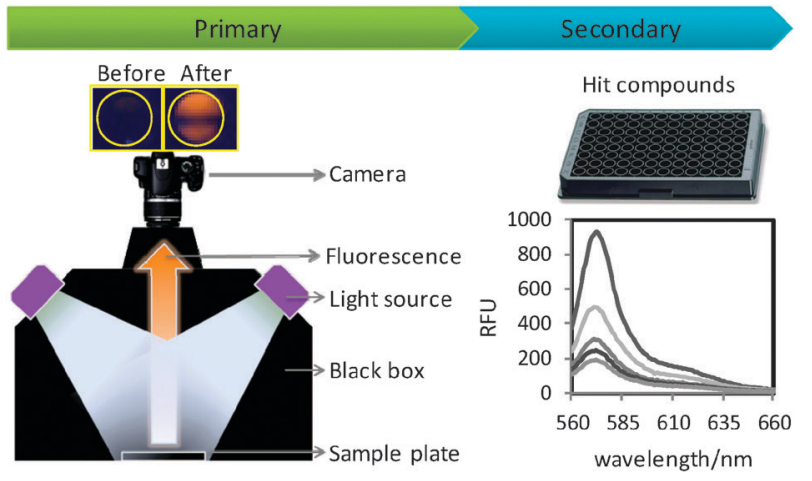

Fig. 2 Schematic work flow of primary and secondary screening in GBL sensor development.

similarity of containing a hydroxyl group at either the para- or the meta-position of the phenyl ring (Fig. 3a). Postulating that a hydroxyl group may play a prominent role in the interaction with GBL, we further synthesized the derivatives with a di-hydroxyl group. Interestingly, the 3,5-dihydroxyl compound (named Green Date) showed even better response to GBL than the previously selected 5 hits, while the 3,4-dihydroxyl compound exhibited poor stability in aqueous solution. Green Date (Fig. 3a) was then selected as the final GBL fluorescent sensor for further study.

Green Date has an absorption and an emission maximum at 569 and $582 \mathrm{~nm}$, respectively, with a quantum yield of 0.05 in water. It exhibited more than 2-fold fluorescent increase with $10 \mathrm{mg} \mathrm{mL}^{-1}$ GBL in water, and has a detection limit of $3 \mathrm{mg} \mathrm{mL}^{-1}$ (Fig. 3b). The fluorescence intensity of Green Date showed a linear increase with respect to the concentration of GBL within the 0 to $100 \mathrm{mg} \mathrm{mL}^{-1}$ range. As GBL is usually dosed in drinks, especially alcohols, we next
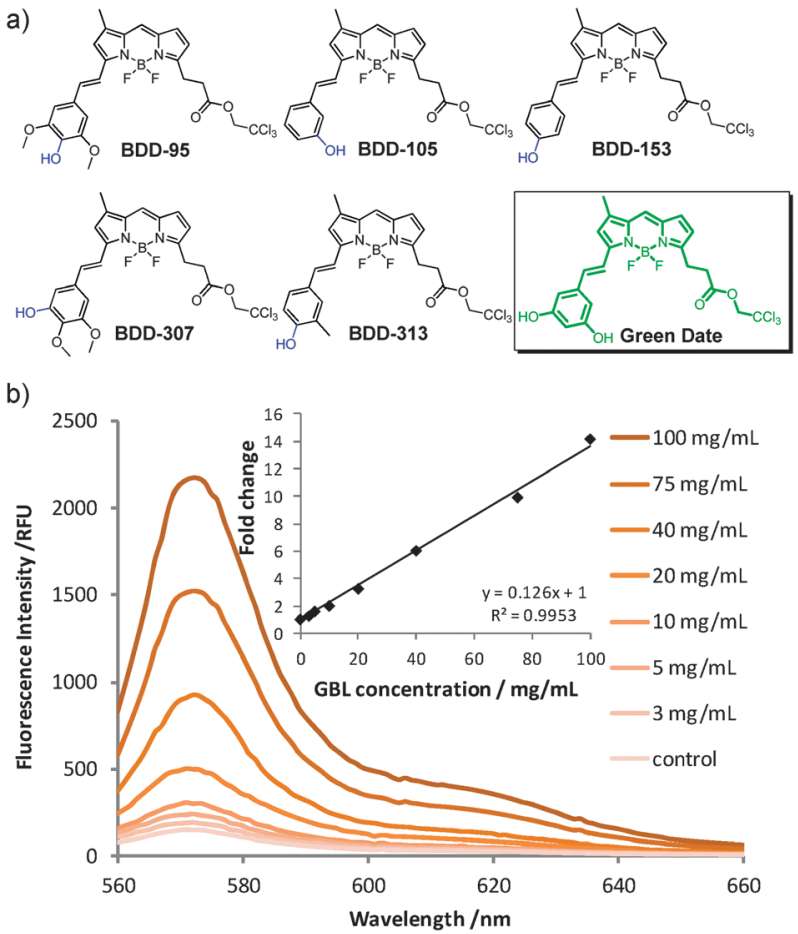

Fig. 3 (a) Structures of the 5 hit compounds for GBL and Green Date. (b) Fluorescent spectra of Green Date $(10 \mu \mathrm{M})$ after incubation with different concentrations of GBL. (inner) Linear correlation of fold change in fluorescence versus concentration of GBL. evaluated the pH-dependence and ethanol-effect of Green Date. Consistent fluorescent response to GBL was observed within the $\mathrm{pH}$ range of 2 to 11 (Fig. S4, ESI + ), indicating that Green Date can be used under both acidic and basic conditions. Low percentage of ethanol (i.e., up to 10\%) did not significantly affect the linear fluorescent enhancement of Green Date to GBL (Fig. S5, ESI $†$ ), showing that it can also detect GBL in alcoholic drinks.

In order to test the efficiency of Green Date as a convenient GBL detection kit, we tested various real drink samples spiked with GBL. Several beverages representing alcoholic, non-alcoholic, coloured and colourless drinks were selected for this test. For the development of a visual detection kit of GBL by Green Date in real samples, we introduce an extraction method. This extraction method not only helps to remove the interfering coloured components from drinks but also concentrate GBL. Drink samples $(2 \mathrm{~mL})$ were extracted with $2 \mathrm{~mL}$ dichloromethane. The organic layer was then separated, airdried, and re-suspended in $100 \mu \mathrm{L}$ water containing Green Date (Fig. 4a). This method can concentrate GBL more than 10 times its original concentration in drinks, and also other interfering components in the drinks (e.g., ethanol, coloured materials) were not extracted by dichloromethane. Although the recovery yield from different drink samples varies, it was clear that Green Date showed very different fluorescence intensity to the drinks with and without GBL. Furthermore, the safety of a drink could be simply visualized using a green laser pointer after the extraction method. The green laser light can pass through the sample containing no GBL, while orange fluorescence is turned on when the sample contains GBL (Fig. 4b). These results illustrated that Green Date is able to detect GBL visually in various drinks after a simple extraction method.

The mechanism of the interaction between Green Date and GBL was further explored. The fact that hydrophobic fluorescent molecules stack together in polar solvents to minimize contact with water and hence diminished the fluorescence is well known as static quenching. ${ }^{18}$ As Green Date is a hydrophobic BODIPY dye, we first characterize the dynamic particle size of Green Date in aqueous solution using a light scattering experiment. As expected, $10 \mu \mathrm{M}$ Green Date aqueous solution shows an average dynamic particle diameter of $64.82 \mathrm{~nm}$. This large size of diameter correlates with the static quenching theory. It also explained why Green Date exhibited high quantum yield in organic solvents (i.e., 0.80 in DMSO), while much lower yield in aqueous solution (i.e., 0.05 in water).
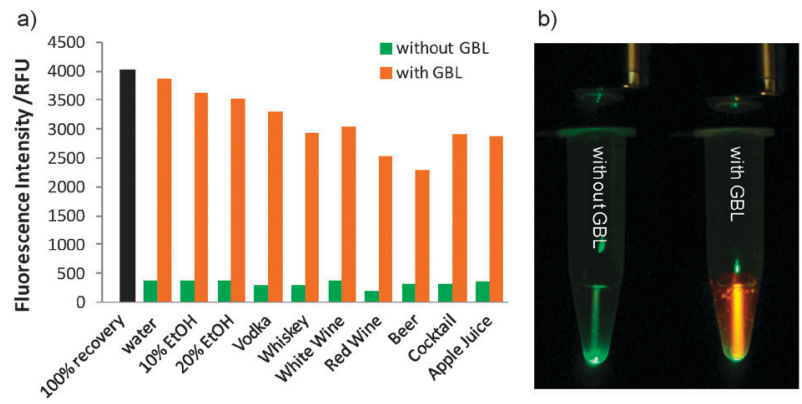

Fig. 4 (a) Fluorescent response of Green Date $(10 \mu \mathrm{M})$ to different drink samples after the extraction method (GBL concentration: $10 \mathrm{mg} \mathrm{mL}^{-1}, 100 \%$ recovery is the theoretical calculation assuming $100 \%$ GBL was extracted), (b) a picture of extraction samples of apple juice with and without GBL containing $50 \mu \mathrm{M}$ Green Date under irradiation using a green laser pointer. 

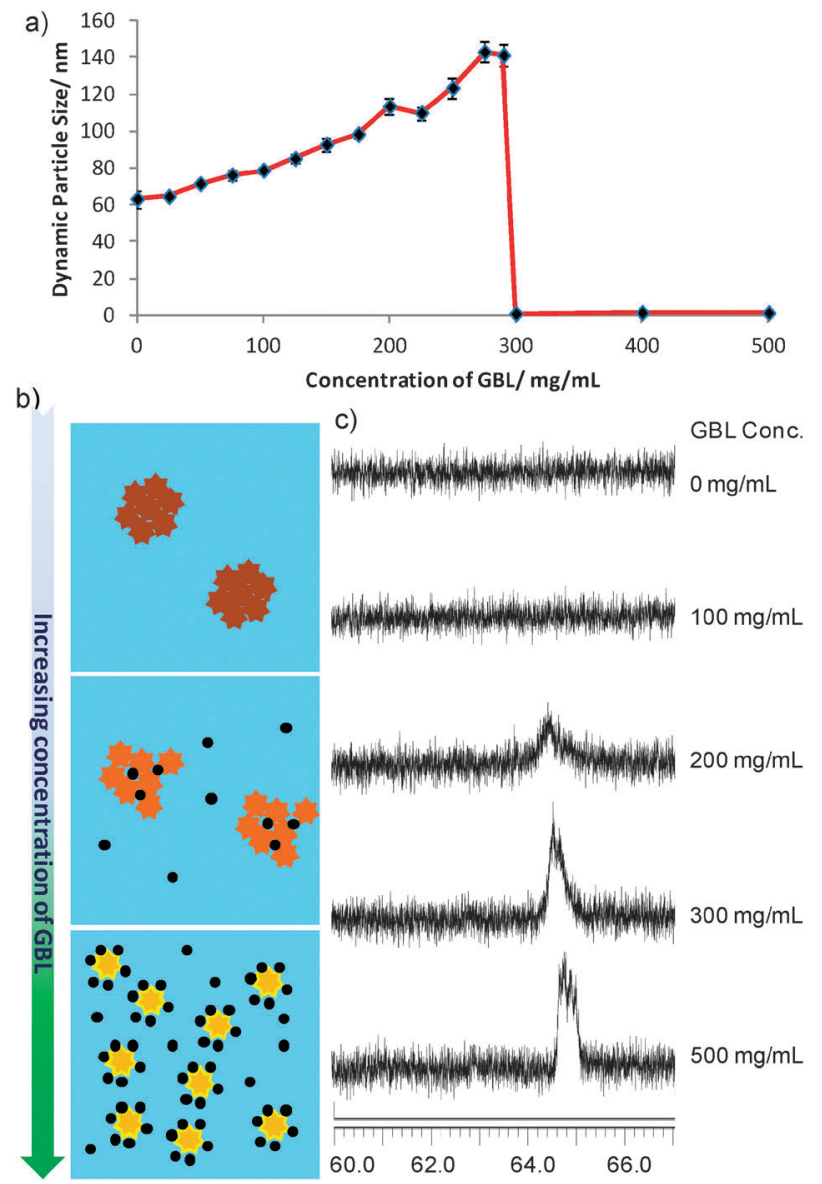

Fig. 5 (a) Dynamic particle size of Green Date with different concentrations of $\mathrm{GBL}$ in water solution. (b) Schematic explanation of the proposed mechanism of interaction between Green Date and GBL (star: Green Date molecule; black ball: $\mathrm{GBL}$ molecule). (c) ${ }^{19} \mathrm{~F}$ NMR spectrum of Green Date $\mathrm{D}_{2} \mathrm{O}$ solution (10 mM with $5 \%$ DMSO- $_{6}$ ) with different concentrations of GBL.

However, with the increasing concentration of GBL in water solution, the dynamic particle size of Green Date obviously increased up to some extent (i.e., around $140 \mathrm{~nm}$ at $290 \mathrm{mg} \mathrm{mL}^{-1}$ of GBL), and then reduced to around $1 \mathrm{~nm}$ (i.e., at $300 \mathrm{mg} \mathrm{mL}^{-1}$ of GBL) (Fig. 5a). Similar disassembly-driven fluorescence turn-on for fluorophores has already been reported by Hamachi and co-workers. ${ }^{19}$ Based on this information, we envisioned the mechanism of the interaction between Green Date and GBL as shown in Fig. 5b. Relatively hydrophobic GBL molecules entered into the stacked nanoparticles of Green Date in aqueous solutions, which enlarged the particle size, and simultaneously reduced the static quenching effect of Green Date to turn on the fluorescence. As the percentage of GBL increased in solution, Green Date molecules in the stacked nanoparticles disassembled, and large dynamic nanoparticles decreased in the solution, hence the fluorescence of Green Date was turned on. Additionally, ${ }^{19} \mathrm{~F}$ NMR signal turn off-on with assembly and disassembly of molecules has also been reported. ${ }^{20}$ This can be explained by the assumption that the ${ }^{19} \mathrm{~F}$ NMR signal is broadened and attenuated when the molecules assemble into aggregates and recovers upon their disassembly. To support our assumption, we also carried out the ${ }^{19} \mathrm{~F}$ NMR experiment of Green Date aqueous samples (10 mM with 5\% DMSO) with different percentages of GBL. The result shows that the ${ }^{19} \mathrm{~F}$ signal of Green Date was completely silent in the absence or with low percentage of GBL. With increasing amount of GBL, ${ }^{19} \mathrm{~F}$ signals appeared and became sharper (Fig. $5 \mathrm{c}$ ). This also approved the aggregate-disassembled mechanism between Green Date and GBL.

In summary, in order to discover a fluorescent sensor for the illicit date rape drug, GBL, we performed high-throughput screening using in-house 5120 compounds, and identified Green Date as a novel GBL sensor. Green Date showed high fluorescence response to GBL. It was later proved to be working under various $\mathrm{pH}$ conditions and in up to $10 \%$ EtOH. Furthermore, Green Date is able to detect the existence of GBL in different kinds of drink samples after a simple extraction method with obvious colour change under the irradiation using a green laser pointer. The mechanism of the interaction between GBL and Green Date was explored using light scattering and ${ }^{19} \mathrm{~F}$ NMR experiments. This discovery will improve the protection against DFSA. With an efficient probe Green Date for visual detection of GBL in drink, we are further working to develop a practical, cheap and rapid detection kit for GBL.

This study was supported by an intramural funding from A*STAR (Agency for Science, Technology and Research, Singapore) Biomedical Research Council and a Singapore Ministry of Education Academic Research Fund Tier 2 (MOE2010-T2-1-025).

\section{Notes and references}

1 S. C. Bishop, M. Lerch and B. R. McCord, Forensic Sci. Int., 2004, 141, 7-15.

2 R. H. Schwartz, R. Milteer and M. A. LeBeau, South. Med. J., 2000, 93, 558-561.

3 K. L. Nicholson and R. L. Balster, Drug Alcohol Depend., 2001, 63, 1-22.

4 J. O. Savino and B. E. Turvey, Rape Investigation Handbook, Academic Press, Waltham, MA, 2nd edn, 2011, p. 335.

5 P. E. Mason and W. P. Kerns 2nd, Acad. Emerg. Med., 2002, 9, 730-739.

6 J. Lettieri and H. L. Fung, Res. Commun. Chem. Pathol. Pharmacol., 1978, 22, 107-118.

7 M. Shannon and L. S. Quang, Pediatr. Emerg. Care, 2000, 16, 435-440.

8 A. D. Brailsford, D. A. Cowan and A. T. Kicman, J. Anal. Toxicol., 2012, 36, 88-95.

9 P. Palatini, L. Tedeschi, G. Frison, R. Padrini, R. Zordan, R. Orlando, L. Gallimberti, G. L. Gessa and S. D. Ferrara, Eur. J. Clin. Pharmacol., 1993, 45, 353-356.

10 (a) D. M. Roberts, M. W. Smith, M. Gopalakrishnan, G. Whittaker and R. O. Day, Ann. Emerg. Med., 2011, 58, 83-85; (b) M. E. Liechti, I. Kunz, P. Greminger, R. Speich and H. Kupferschmidt, Drug Alcohol Depend., 2006, 81, 323-326.

$11 \mathrm{http}: / /$ www.drinksafetech.com/.

$12 \mathrm{http}: / /$ www.drinkdetective.com/science.asp.

13 M. Bradley and A. Lewis, US Pat., 0039346 A1, 2011.

14 R. B. Palmer, Toxicol. Rev., 2004, 23, 21-31.

15 (a) J. S. Lee, Y. K. Kim, M. Vendrell and Y. T. Chang, Mol. BioSyst., 2009, 5, 411-421; (b) M. Vendrell, J. S. Lee and Y. T. Chang, Curr. Opin. Chem. Biol., 2010, 14, 383-389.

16 C. T. Lesar, J. Decatur, E. Lukasiewicz and E. Champeil, Forensic Sci. Int., 2011, 212, e40-e45.

17 D. Zhai, S. C. Lee, M. Vendrell, L. P. Leong and Y. T. Chang, ACS Comb. Sci., 2012, 14, 81-84.

18 J. R. Lakowicz, Principles of Fluorescence Spectroscopy, Kluwer Academic/Plenum, New York, 3rd edn, 1999, p. 282.

19 (a) K. Mizusawa, Y. Ishida, Y. Takaoka, M. Miyagawa, S. Tsukiji and I. Hamachi, J. Am. Chem. Soc., 2010, 132, 7291-7293; (b) K. Mizusawa, Y. Takaoka and I. Hamachi, J. Am. Chem. Soc., 2012, 134, 13386-13395.

20 Y. Takaoka, T. Sakamoto, S. Tsukiji, M. Narazaki, T. Matsuda, H. Tochio, M. Shirakawa and I. Hamachi, Nat. Chem., 2009, 1, 557-561. 